\title{
SMOOTH SOLUTIONS OF SYSTEMS OF QUASILINEAR PARABOLIC EQUATIONS
}

\author{
Alain Bensoussan ${ }^{1}$ And Jens Frehse ${ }^{2}$
}

\begin{abstract}
We consider in this article diagonal parabolic systems arising in the context of stochastic differential games. We address the issue of finding smooth solutions of the system. Such a regularity result is extremely important to derive an optimal feedback proving the existence of a Nash point of a certain class of stochastic differential games. Unlike in the case of scalar equation, smoothness of solutions is not achieved in general. A special structure of the nonlinear Hamiltonian seems to be the adequate one to achieve the regularity property. A key step in the theory is to prove the existence of Hölder solution.
\end{abstract}

Mathematics Subject Classification. 35XX, 49XX.

Received January 29, 2002.

\section{INTRODUCTION}

In this paper, we consider diagonal parabolic systems arising in the context of stochastic differential games. These systems are of the form

$$
\frac{\partial}{\partial t} u_{\nu}-\sum_{i, j=1}^{n} \frac{\partial}{\partial x_{i}}\left(a_{i j}(t, x) \frac{\partial}{\partial x_{j}} u_{\nu}\right)=H_{\nu}(x, u, \nabla u), \quad \nu=1, \ldots, N
$$

where the "Hamiltonians" $H_{\nu}$ have quadratic growth in $\nabla u$. In application to game theory, $N$ corresponds to the number of players. The aim is to prove global existence and regularity results under the assumptions of uniform ellipticity for $a_{i j}$ and special structure conditions for $H_{\nu}$, say

$$
\left|H_{\nu}(x, u, p)\right| \leq K\left|p_{\nu}\right||p|+K \sum_{\mu<\nu}\left|p_{\mu}\right|^{2}+K
$$

provided some $L^{\infty}$-estimate is available, for which in turn, simple conditions are presented.

Keywords and phrases: Parabolic equations, quasilinear, game theory, regularity, Stochastic optimal control, smallness condition, specific structure, maximum principle, Green function, Hamiltonian.

1 University Paris-Dauphine and CNES, France.

${ }^{2}$ Institut für Angewandte Mathematik der Universität Bonn, Germany. 
The theory of scalar parabolic equations (1.1) for the right-hand side having general quadratic growth is well understood since $C^{\alpha}$-estimates are available without smallness conditions for the growth-factor $a$,

$$
|H(x, u, \nabla u)| \leq a|\nabla u|^{2}+K
$$

$c f$. the book [15]. For examples of scalar parabolic equations arising from stochastic optimal control, $c f$. Bensoussan and Lions [8].

For parabolic systems with "large" growth factor, $C^{\alpha}$-estimates can not be achieved in general. Struwe presented an example of the same type of system as (1.1), where a discontinuous weak solution develops from the zero solution!

$C^{\alpha}$-regularity and $C^{\alpha}$-bounds follow from a smallness condition for the growth-factor $a$, infact $|a|<\|u\|_{\infty} \lambda$, $\lambda$ ellipticity constant $c f$., Struwe [16]. However, for applications in the theory of stochastic games such a condition for the growth factor $a$ is not acceptable since the ellipticity constant may be very small compared to the growth-factor. So, in order to avoid smallness conditions the authors [4] assumed the "specific structure condition" (1.2) in the case of two players and obtained $C^{\alpha}$-estimates and hence $L^{q}\left(W^{2, q}\right)$-solutions, $q<\infty$, for (1.1). In fact [4] contains also cases with $N$ players, $N \geq 3$, but not with the "simple" assumption (1.2). In $[3,5-7]$ and [2] examples of games mainly with two players, where (1.2) holds, are presented and can be extended to the parabolic case.

Note, that a similar discussion concerning the growth factor holds for the elliptic analogue; references for $C^{\alpha}$-estimates and -regularity under rather optimal smallness condition are $[13,17]$.

Note further, that, without $C^{\alpha}$-estimates, in general it is false that a sequence of (possibly approximate) solutions to (1.2), which is bounded in $L^{\infty}\left(L^{2}\right) \cap L^{2}\left(H^{1}\right)$, has a subsequence which converges strongly in $L^{2}\left(H^{1}\right)$. This type of convergence allows to pass to the limit in the terms $\int H_{\nu} \varphi \mathrm{d} x$. For a counter example in the elliptic case, see the example [11]. Therefore, for systems (1.1) and its elliptic analogue the well known theory of Leray and Lions [14] can not be applied. This explains the difficulty of the analysis.

The analysis for obtaining $C^{\alpha}$-estimates (and hence $W^{2, p}$-estimates and existence theorems) for the above system with $N>2$ and the specific structure condition (1.2) is the main contribution of this paper.

In the elliptic analogue, the corresponding case of three and four players has been worked out in [12]. In fact, the methods $[3,4,7]$ and [2] are extended to the general case of $N$ players and the parabolic situation. Note that the main results of this paper apply to the elliptic case (assume $u(t, x)$ is constant in time) and that, also in the elliptic case, the results are new.

\section{Setting of the PRoblem And Statement of Results}

\subsection{Preliminary setting and assumptions}

Let $\Omega$ be a smooth bounded domain of $\mathbb{R}^{n}$ and $Q=\Omega \times(0, T)$. We write

$$
\Sigma=\partial \Omega \times(0, T)
$$

Let $a_{i j}(x, t), i, j=1, \ldots, n$ be given functions satisfying

$$
a_{i j} \in C^{1}(\bar{Q})
$$

$$
a_{i j} \xi_{j} \xi_{i} \geq \alpha|\xi|^{2} \quad \forall \xi \in R^{n}, \quad \alpha>0
$$


We define the family of 2nd order elliptic operators in divergence form

$$
A \equiv A(t)=-\frac{\partial}{\partial x_{i}} a_{i j} \frac{\partial}{\partial x_{j}}
$$

with Dirichlet boundary conditions.

We consider a vector function $H(x, t ; \lambda, p)$ from $\mathbb{R}^{n} \times[0, T] \times \mathbb{R}^{N} \times \mathbb{R}^{N n}$ into $\mathbb{R}^{N}$, such that

$$
H \text { is measurable, and continuous on } \lambda, p \text {. }
$$

We denote by $H_{\nu}, \nu=1, \ldots, N$, the components of the vector $H$. In fact, we shall make use of linear manipulations on the components of $H$ as follows.

Let $\Gamma$ be an $N \times N$-matrix which is invertible, then to $\Gamma$ we associate the transform of $H$, denoted $H^{\Gamma}$ and defined by

$$
H^{\Gamma}(x, t ; \lambda, p)=\Gamma H\left(x, t ; \Gamma^{-1} \lambda, \Gamma^{-1} p\right) .
$$

We shall say that a matrix $\Gamma$ satisfies the maximum principle, whenever

$$
\text { if } \Gamma \lambda \geq 0, \quad \text { then } \lambda \geq 0 \text {. }
$$

We shall make use of three alternative sets of assumptions. These conditions will us give $L^{\infty}$-estimates.

$$
\left|H_{\nu}(x, t, \lambda, p)\right|_{p_{\nu}=0} \leq K
$$

or, alternatively, the following two conditions (2.8) and (2.9):

$$
\sum_{\nu} H_{\nu}(x, t ; \lambda, p) \geq-\gamma \text { for those } p \text { such that } \sum_{\nu=1}^{N} p_{\nu}=0
$$

and

$$
\left.H_{\nu}^{\Gamma}(x, t ; \lambda, p)\right|_{p_{\nu}=0} \leq \gamma_{\nu} \text { for some matrix } \Gamma \text { satisfying the maximum principle }
$$

or, alternatively, the following conditions (2.10) and (2.11):

$$
\sum_{\nu} H_{\nu}(x, t ; \lambda, p) \leq \gamma \text { for those } p \text { such that } \sum_{\nu=1}^{N} p_{\nu}=0
$$

and

$$
\left.H_{\nu}^{\Gamma}(x, t ; \lambda, p)\right|_{p_{\nu}=0} \geq-\gamma_{\nu} \text {. for some matrix } \Gamma \text { which satisfies the maximum principle. }
$$

The last two pairs of conditions increases the applicability of the theory to stochastic differential games considerably, cf. [2,5] and [7].

The next assumption specifies what we shall call the specific structure, namely there exists a matrix $\Gamma$ such that

$$
\left|H_{\nu}^{\Gamma}(x, t ; \lambda, p)\right| \leq K_{\nu}|p|\left|p_{\nu}\right|+\sum_{\mu=1}^{\nu} K_{\mu}^{\nu}\left|p_{\mu}\right|^{2}+k_{\nu}(x, t), \quad \nu=1, \ldots, N-1
$$


where $K_{\nu}, K_{\mu}^{\nu}$ are positive constants and $k_{\nu}$ positive functions $\in L^{q}(Q)$, with $q>\frac{n}{2}+1$. Moreover

$$
\left|H_{N}^{\Gamma}(x, t ; \lambda, p)\right| \leq K_{N}|p|^{2}+k_{N}(x, t)
$$

with a positive constant $K_{N}$, a positive function $k_{N} \in L^{q}(Q)$, with $q>\frac{n}{2}+1$.

Without loss of generality, the special structure $(2.12,2.13)$ can be formulated as follows, dropping $\Gamma$ to simplify the notation

$$
H_{\nu}(x, t ; \lambda, p)=Q_{\nu}(x, t ; \lambda, p) p_{\nu}+H_{\nu}^{0}(x, t ; \lambda, p), \quad \nu=1, \ldots, N
$$

with

$$
\begin{aligned}
& Q_{\nu} \text { measurable, continuous in } \lambda, p, \text { for } p_{\nu} \neq 0, \\
& \left|Q_{\nu}(x, t ; \lambda, p)\right| \leq K_{\nu}|p| \quad \nu=1, \ldots,=N-1, \\
& Q_{N}=Q_{N-1},
\end{aligned}
$$

$H_{\nu}^{0}$ measurable, continuous in $\lambda, p, \nu=1, \ldots, N-1$,

$$
\left|H_{\nu}^{0}(x, t ; \lambda, p)\right| \leq \sum_{\mu=1}^{\nu} K_{\mu}^{\nu}\left|p_{\mu}\right|^{2}+k_{\nu}(x, t), \nu=1, \ldots, N
$$

where we have defined

$$
K_{\mu}^{N}=K_{N}+\frac{K_{N}-1}{2}, \mu=1, \ldots, N-1 ; \quad K_{N}^{N}=K_{N}+K_{N-1} .
$$

Indeed, we set, for $\nu=1, \ldots, N-1$

$$
\sigma_{\nu}(x, t ; \lambda, p)=\frac{H_{\nu}(x, t ; \lambda, p)}{K_{\nu}|p|\left|p_{\nu}\right|+\sum_{\mu=1}^{\nu} K_{\mu}^{\nu}\left|p_{\mu}\right|^{2}+k_{\nu}(x, t)}
$$

and successively

$$
\begin{aligned}
& Q_{\nu}(x, t ; \lambda, p)=K_{\nu} \sigma_{\nu}(x, t ; \lambda, p)|p| \frac{p_{\nu}}{\left|p_{\nu}\right|}, \quad \nu=1, \ldots, N-1, \\
& Q_{N}=Q_{N-1} . \\
& H_{\nu}^{0}(x, t ; \lambda, p)=H_{\nu}(x, t ; \lambda, p)-Q_{\nu}(x, t ; \lambda, p) p_{\nu}, \quad \nu=1, \ldots, N
\end{aligned}
$$

and (2.14-2.16) are satisfied.

\subsection{The problem}

Let be given

$$
u^{0} \in\left(W_{0}^{1, \infty}(\Omega)\right)^{N},
$$


we look for a function $u$ such that

$$
\begin{array}{cc}
u \in\left(L^{q}\left(0, T ; W_{0}^{2, q}(\Omega)\right)\right)^{N}, & \frac{\partial u}{\partial t} \in\left(L^{q}(Q)\right)^{N}, \\
\frac{\partial u}{\partial t}+A(t) u=H(x, t, u, D u), & \forall x, t, \\
u(0)=u^{0},\left.\quad u\right|_{\Sigma}=0 . &
\end{array}
$$

Thanks to the transformation $\Gamma$ we can imbed the problem (2.21-2.23) into a family of problems as follows:

If $u$ is a solution of $(2.21-2.23)$ then set

$$
z=\Gamma u
$$

and clearly $z$ is a solution of the same problem, with $H$ replaced by $H^{\Gamma}$, and $u^{0}$ by $\Gamma u^{0}$.

We state the following:

Theorem 2.1. Let the smoothness conditions (2.1, 2.4, 2.20) on the data and the ellipticity condition (2.2) be satisfied. Assume the specific growth conditions (2.12, 2.13) on the Hamiltonians and finally the structure conditions (2.8, 2.9) or, alternatively (2.10, 2.11) instead of (2.8, 2.9). Then there exists a global solution of the parabolic system (2.21-2.23).

By "global" we mean that no smallness assumption for $T$ is assumed.

Remark 2.1. The structure conditions $(2.8,2.9)$ resp. $(2.10,2.11)$ are "responsible" for the $L^{\infty}$-estimate, the structure conditions $(2.12,2.13)$ for the step from $L^{\infty}$ to $C^{\alpha} \cap H^{1}$.

\section{FiRst a PRIORI ESTIMATES}

\subsection{A fundamental inequality}

We consider a solution of $(2.21-2.23)$. We shall write

$$
\rho=\|u\|_{\left(L^{\infty}(Q)\right)^{N}} .
$$

To the solution $u$ we associate any constant vector $c$ such that

$$
\|c\| \leq \rho
$$

and we write

$$
\tilde{u}=u-c .
$$

Let also

$$
\begin{gathered}
\psi \geq 0, \quad \psi \in L^{2}\left(0, T, H^{1}(\Omega)\right), \quad \psi \in L^{\infty}(Q), \\
\left.\psi(x, t)\right|_{\partial \Omega}=0, \quad \forall t \in(0, T) \text { if } c \neq 0 .
\end{gathered}
$$


We introduce the notation

$$
\beta(x)=\mathrm{e}^{x}-x-1
$$

and the map $X(s): \mathbb{R}^{N} \rightarrow \mathbb{R}^{N}$, defined backwards by the formulas

$$
\begin{aligned}
X_{N}(s) & =\exp \left[\beta\left(\gamma_{N} s_{N}\right)+\beta\left(-\gamma_{N} s_{N}\right)\right], \\
X_{\nu}(s) & =\exp \left[\beta\left(\gamma_{\nu} s_{\nu}\right)+\beta\left(-\gamma_{\nu} s_{\nu}\right)+X_{\nu+1}\right], \quad \nu=1, \ldots, N-1,
\end{aligned}
$$

where $\gamma_{\nu}$ are positive constants and $s=\left(s_{1}, \ldots, s_{N}\right)$.

We note that

$$
\frac{\partial X_{\nu}}{\partial s_{\mu}}=\left\{\begin{array}{l}
0 \text { if } \mu<\nu \\
\gamma_{\mu} X_{\nu} \ldots X_{\mu}\left(\beta^{\prime}\left(\gamma_{\mu} s_{\mu}\right)-\beta^{\prime}\left(-\gamma_{\mu} s_{\mu}\right)\right) \text { if } \mu \geq \nu .
\end{array}\right.
$$

We call

$$
X(x, t)=X(\tilde{u}(x, t))
$$

hence, clearly

$$
\begin{aligned}
& D X_{\nu}=\sum_{\mu=\nu}^{N} \gamma_{\mu} X_{\nu} \ldots X_{\mu}\left(\beta^{\prime}\left(\gamma_{\mu} \tilde{u}_{\mu}\right)-\beta^{\prime}\left(-\gamma_{\mu} \tilde{u}_{\mu}\right)\right) D u_{\mu} \\
& \frac{\partial X_{\nu}}{\partial t}=\sum_{\mu=\nu}^{N} \gamma_{\mu} X_{\nu} \ldots X_{\mu}\left(\beta^{\prime}\left(\gamma_{\mu} \tilde{u}_{\mu}\right)-\beta^{\prime}\left(-\gamma_{\mu} \tilde{u}_{\mu}\right)\right) \frac{\partial u_{\mu}}{\partial t}
\end{aligned}
$$

from which we have the estimates

$$
\begin{aligned}
& |D X| \leq c(\rho)|\tilde{u}||D u|, \\
& \left|\frac{\partial X}{\partial t}\right| \leq c(\rho)|\tilde{u}|\left|\frac{\partial u}{\partial t}\right|,
\end{aligned}
$$

where in the sequel $c(\rho)$ will denote a constant (not precised explicitly) depending only on $\rho$ (this assumes that the constants $\gamma_{\nu}$ are defined only as functions of $\rho$, which will be the case as shown below).

Note also, that thanks to formulas (3.7)

$$
|X-X(0)| \leq c(\rho)|\tilde{u}|^{2},
$$

where $X(0)$ is the value of $X(s)$, for $s=0$.

Moreover,

$$
X \geq X(0) \geq 1 .
$$

We state the following:

Proposition 3.1. If we assume(2.1, 2.2, 2.12, 2.13, 2.20), and if we have a solution u of (2.21-2.23), then there exist $\gamma_{\nu}(\rho), c(\rho)$, such that, for any constant vector $c$ subject to (3.3), and any $\psi$ such that (3.4) holds, 
one has

$$
\int_{Q} \psi \frac{\partial X_{1}}{\partial t} \mathrm{~d} x \mathrm{~d} t+\int_{Q} a_{i j} \frac{\partial X_{1}}{\partial x_{j}} \frac{\partial \psi}{\partial x_{j}} \mathrm{~d} x \mathrm{~d} t+\alpha \int_{Q} \psi|D u|^{2} \mathrm{~d} x \mathrm{~d} t \leq c(\rho) \int_{Q} \psi \sum_{\nu=1}^{N} k_{\nu} \mathrm{d} x \mathrm{~d} t .
$$

Proof of Proposition 3.1. We take as a test function in (2.22)

$$
v_{\nu}=\psi \gamma_{\nu}\left(\beta^{\prime}\left(\gamma_{\nu} \tilde{u}_{\nu}\right)-\beta^{\prime}\left(-\gamma_{\nu} \tilde{u}_{\nu}\right)\right) \prod_{\mu=1}^{\nu} X_{\mu}
$$

then from (3.10) it is easy to check that

$$
\sum_{\nu=1}^{N} \frac{\partial u_{\nu}}{\partial t} v_{\nu}=\psi \frac{\partial X_{1}}{\partial t}
$$

Similarly,

$$
\begin{aligned}
\int_{Q} A(t) u_{\nu} v_{\nu} \mathrm{d} x \mathrm{~d} t= & \int_{Q} a_{i j} \frac{\partial u_{\nu}}{\partial x_{j}} \frac{\partial \psi}{\partial x_{i}} \gamma_{\nu}\left(\beta^{\prime}\left(\gamma_{\nu} \tilde{u}_{\nu}\right)-\beta^{\prime}\left(-\gamma_{\nu} \tilde{u}_{\nu}\right)\right) \prod_{\mu=1}^{\nu} X_{\mu} \mathrm{d} x \mathrm{~d} t \\
& +\int_{Q} a_{i j} \frac{\partial u_{\nu}}{\partial x_{j}} \psi \gamma_{\nu}^{2}\left(\beta^{\prime \prime}\left(\gamma_{\nu} \tilde{u}_{\nu}\right)+\beta^{\prime \prime}\left(-\gamma_{\nu} \tilde{u}_{\nu}\right)\right) \frac{\partial u_{\nu}}{\partial x_{i}} \prod_{\mu=1}^{\nu} X_{\mu} \mathrm{d} x \mathrm{~d} t \\
& +\int_{Q} a_{i j} \frac{\partial u_{\nu}}{\partial x_{j}} \psi \gamma_{\nu}\left(\beta^{\prime}\left(\gamma_{\nu} \tilde{u}_{\nu}\right)-\beta^{\prime}\left(-\gamma_{\nu} \tilde{u}_{\nu}\right)\right) \frac{\partial}{\partial x_{i}} \prod_{\mu=1}^{\nu} X_{u} \mathrm{~d} x \mathrm{~d} t \\
= & I+I I+I I I,
\end{aligned}
$$

and as easily seen

$$
\begin{aligned}
& I=\int_{Q} a_{i j} \frac{\partial \psi}{\partial x_{i}} \frac{\partial X_{1}}{\partial x_{j}} \mathrm{~d} x \mathrm{~d} t \\
& I I \geq \alpha \int_{Q} \psi \gamma_{\nu}^{2}|D u|^{2}\left(\mathrm{e}^{\gamma_{\nu} \tilde{u}_{\nu}}+\mathrm{e}^{-\gamma_{\nu} \tilde{u}_{\nu}}\right) \prod_{\mu=1}^{\nu} X_{\mu} \mathrm{d} x \mathrm{~d} t \\
& I I I=\int_{Q} a_{i j} \frac{\partial F_{\nu}}{\partial x_{j}} \frac{\partial F_{\nu}}{\partial x_{i}} \psi \prod_{\mu=1}^{\nu} X_{\mu} \mathrm{d} x \mathrm{~d} t
\end{aligned}
$$

where $F_{\nu}=\log X_{\nu}$

Now

$$
\begin{aligned}
\sum_{\nu=1}^{N-1} \int_{Q} Q_{\nu} D u_{\nu} v_{\nu} \mathrm{d} x \mathrm{~d} t & =\sum_{\nu=1}^{N-1} \int_{Q} \psi Q_{\nu}\left(D F_{\nu}-D X_{\nu+1}\right) \prod_{\mu=1}^{\nu} X_{\mu} \mathrm{d} x \mathrm{~d} t \\
& =\sum_{\nu=1}^{N-1} \int_{Q} \psi\left(Q_{\nu}-Q_{\nu-1}\right) D F_{\nu} \prod_{\mu=1}^{\nu} X_{\mu} \mathrm{d} x \mathrm{~d} t-\int_{Q} \psi Q_{N-1} D F_{N} \prod_{\mu=1}^{N} X_{\mu} \mathrm{d} x \mathrm{~d} t
\end{aligned}
$$


where we have set $Q_{0}=0$. Since $Q_{N}=Q_{N-1}$, it follows that

$$
\sum_{\nu=1}^{N-1} \int_{Q} Q_{\nu} D u_{\nu} v_{\nu} \mathrm{d} x \mathrm{~d} t=\sum_{\nu=1}^{N-1} \int_{Q} \psi \tilde{Q}_{\nu} D F_{\nu} \prod_{\mu=1}^{\nu} X_{\mu} \mathrm{d} x \mathrm{~d} t
$$

with

$$
\tilde{Q}_{\nu}=Q_{\nu}-Q_{\nu-1}, \quad \nu=1, \ldots, N-1 .
$$

Collecting results and performing additional majorations yields

$$
\begin{aligned}
\int_{Q} \psi \frac{\partial X_{1}}{\partial t} \mathrm{~d} x \mathrm{~d} t & +a_{i j} \frac{\partial \psi}{\partial x_{i}} \frac{\partial X_{1}}{\partial x_{j}} \mathrm{~d} x \mathrm{~d} t+\alpha \sum_{\nu=1}^{N} \int_{Q} \psi \gamma_{\nu}^{2}\left|D u_{\nu}\right|^{2}\left(\mathrm{e}^{\gamma u_{\nu}}+\mathrm{e}^{-\gamma u_{\nu}}\right) \prod_{\mu=1}^{\nu} \chi_{\mu} \mathrm{d} x \mathrm{~d} t \leq \sum_{\nu=1}^{N-1} \int_{Q} \psi \frac{a^{-1} \tilde{Q}_{\nu} \cdot \tilde{Q}_{\nu}}{4} \\
& +\sum_{\nu=1}^{N} \int_{Q} \psi H_{\nu}^{0} \gamma_{\nu}\left(\mathrm{e}^{\gamma_{\nu} \tilde{u}_{\nu}}-\mathrm{e}^{-\gamma_{\nu} \tilde{u}_{\nu}}\right) \prod_{\mu=1}^{\nu} X_{\mu} \mathrm{d} x \mathrm{~d} t
\end{aligned}
$$

and thanks to $(2.16)$,

$$
\begin{aligned}
\leq & \sum_{\nu=1}^{N} \int_{Q} \psi\left|D u_{\nu}\right|^{2}\left[\frac{\left\|a^{-1}\right\|}{4} \sum_{\sigma=1}^{N-1}\left(K_{\sigma}+K_{\sigma-1}\right)^{2} \prod_{\mu=1}^{\sigma} X_{\mu}\right. \\
& \left.+\sum_{\sigma=\nu}^{N} \gamma_{\sigma} K_{\nu}^{\sigma}\left|\mathrm{e}^{\gamma_{\sigma} \tilde{u}_{\sigma}}-\mathrm{e}^{-\gamma_{\sigma} \tilde{u}_{\sigma}}\right| \prod_{\mu=1}^{\sigma} X_{\mu}\right] \mathrm{d} x \mathrm{~d} t \\
& +\sum_{\nu=1}^{N} \int_{Q} \gamma_{\nu} \psi k_{\nu}\left|\mathrm{e}^{\gamma_{\nu} \tilde{u}_{\nu}}-\mathrm{e}^{-\gamma_{\nu} \tilde{u}_{\nu}}\right| \prod_{\mu=1}^{\nu} X_{\mu} \mathrm{d} x \mathrm{~d} t .
\end{aligned}
$$

Suppose $\gamma_{\nu}(\rho)$ are chosen so that

$$
\begin{aligned}
\alpha \gamma_{\nu}^{2}-2 \gamma_{\nu} K_{\nu}^{\nu} \geq & \frac{\left\|a^{-1}\right\|}{4}\left[\sum_{\sigma=1}^{\nu}\left(K_{\sigma}+K_{\sigma-1}\right)^{2}\right. \\
& \left.+\sum_{\sigma=\nu+1}^{N-1}\left(K_{\sigma}+K_{\sigma-1}\right)^{2} \sum_{\mu=\nu+1}^{\sigma} X_{\mu}\right]+\sum_{\sigma=\nu+1}^{N} \gamma_{\sigma} K_{\gamma}^{\sigma}\left|\mathrm{e}^{\gamma_{\sigma} \tilde{u}_{\sigma}}-\mathrm{e}^{-\gamma_{\sigma} \tilde{u}_{\sigma}}\right| \prod_{\mu=\nu+1}^{\sigma} X_{\mu}
\end{aligned}
$$

and $c(\rho)$ is such that

$$
\gamma_{\nu}\left|\mathrm{e}^{\gamma_{\nu} \tilde{u}_{\nu}}-\mathrm{e}^{-\gamma_{\nu} \tilde{u}_{\nu}}\right| \prod_{\mu=1}^{\nu} X_{\mu} \leq c(\rho)
$$

Then one obtains (3.15). The constants $\gamma_{\nu}$ depending only on $\rho$, can be defined from the inequalities (3.16) backwards, observing that $X_{\mu}$ can be majorized by a number depending only on $\rho, \gamma_{\mu}, \ldots, \gamma_{N}$. The proof has been completed.

\footnotetext{
${ }^{3}$ We implicitly assume the matrix $a=a_{i j}$ symmetric. If not replace $a$ for $\frac{a+a^{*}}{2}$ in the formula.
} 


\section{2. $\boldsymbol{L}^{\mathbf{2}}\left(\mathbf{0}, \boldsymbol{T} ; \boldsymbol{H}^{\mathbf{1}}\right)$ estimate}

If we pick $c=0$ and $\psi=1$ in (3.15), we obtain

$$
\alpha \int_{Q}|D u|^{2} \mathrm{~d} x \mathrm{~d} t \leq \int_{\Omega} X\left(u^{0}\right) \mathrm{d} x+c(\rho) \int_{Q}\left(\sum_{\nu} k_{\nu}\right) \mathrm{d} x \mathrm{~d} t \leq c(\rho),
$$

and thus an estimate of the norm in $\left(L^{2}\left(0, T ; H_{0}^{1}(\Omega)\right)\right)^{N}$ follows from the $L^{\infty}$ norm of $u$, denoted by $\rho$.

\section{3. $\boldsymbol{L}^{\infty}$ estimate}

We state the

Proposition 3.2. We assume (2.1, 2.2, 2.7) (or (2.8, 2.9)) (or (2.10, 2.11)). If we have a solution u of (2.21-2.23), then there exists a number $\rho$ depending only on the constants $\gamma, \gamma_{\nu}$, the matrix $\Gamma$ and $\left\|u^{0}\right\|_{L^{\infty}}$, so that

$$
\|u\|_{\left(L^{\infty}(Q)\right)^{N}} \leq \rho
$$

Proof. We treat only the second alternative. The proof is typical for the other cases.

Since $u_{t} \in L^{q}, \nabla^{2} u \in L^{q}, q$ large, we have $\nabla u \in C^{\alpha}$. Suppose that the boundary $\partial Q$ contains no minimum point of the function $\varphi=e^{-t} \sum_{\nu} u_{\nu}$. Let $M$ be the set of points where $\varphi$ attains its minimum $m$. $M$ is closed and $\nabla \varphi=0$ and $\nabla \sum_{\nu} u_{\nu}=0$. We have $\sum_{\nu} H_{\nu} \geq-\gamma$ on $M$ (assume $(2.8,2.9)$, to fix ideas). Let $U \subset \subset Q$ be an open neighborhood of $M$ such that $\varphi=m+\varepsilon$ on $\partial U, \varepsilon>0$ small. In addition, we may choose $\operatorname{dist}(\partial U, M)$ so small, that $\sum_{\nu} H_{\nu} \geq-\gamma-\delta$ on $U$. (For simplicity we assume here that $H_{\nu}$ is also continuous in $x$. The general case is treated by approximating $H_{\nu}$.)

$$
\left(\frac{\partial}{\partial t}+A(t)\right) \sum_{\nu} u_{\nu} \geq-\gamma-\delta \text { on } U
$$

and

$$
\left(\frac{\partial}{\partial t}+A(t)\right) \varphi+\varphi \geq-\mathrm{e}^{-t}(\gamma+\delta) \text { on } U
$$

Now, suppose that $m+\varepsilon \leq-\mathrm{e}^{-t}(\gamma+\delta), 0 \leq t \leq T$. Then

$$
\left(\frac{\partial}{\partial t}+A(t)\right) \varphi+(\varphi-(m+\varepsilon)) \geq 0 \text { on } U
$$

and we obtain a contradiction if we test by $\min (\varphi-(m+\varepsilon), 0)$. (Take into account that $\varphi-(m+\varepsilon)=0$ on $\partial U$ and $\nabla \varphi$ cannot be zero a.e. on $U$.) This means that either $m+\varepsilon \geq-\mathrm{e}^{-t}(\gamma+\delta)$ or the minimum is attained at $\partial Q$. In both cases, we arrive at a bound for $\sum_{\nu} u_{\nu}$ from below:

$$
\sum_{\nu} u_{\nu} \geq-c
$$


With similar arguments, we achieve a bound for $u_{\nu}, \nu=1, \ldots, N$ from above. Let $z=\Gamma u$. Then $z$ is a solution of

$$
\begin{aligned}
\left(\frac{\partial}{\partial t}+A(t)\right) z & =H^{\Gamma}(z, D z), \\
\left.z\right|_{\Sigma} & =0 \\
z(x, 0) & =\Gamma u^{0}(x)=z_{0}(x) .
\end{aligned}
$$

Suppose that $\varphi_{\nu}=\mathrm{e}^{-t} z_{\nu}$ does not attain its maximum at the boundary and let $M_{\nu}$ be the set of its maximum points. Then $\nabla \varphi_{\nu}=\nabla z_{\nu}=0$ on $M$ and

$$
\left(\frac{\partial}{\partial t}+A(t)\right) \varphi_{\nu}+\varphi_{\nu} \leq \mathrm{e}^{-t} \gamma_{\nu} \text { on } M_{\nu}
$$

Introducing an adequate neighbourhood $U_{\nu}$ of $M_{\nu}$ as above we obtain

$$
\left(\frac{\partial}{\partial t}+A(t)\right) \varphi_{\nu}+\varphi_{\nu} \leq \mathrm{e}^{-t}\left(\gamma_{\nu}+\delta_{\nu}\right) \text { on } U_{\nu}
$$

and if we suppose that $m_{\nu}=\max \varphi_{\nu}$ and $m_{\nu}-\varepsilon \geq \mathrm{e}^{-\gamma}\left(\gamma_{\nu}+\delta_{\nu}\right)$, we obtain a contradiction by testing with $\max \left(0, \varphi_{\nu}-\left(m_{\nu}-\varepsilon\right)\right)$. This gives a bound for $z_{\nu}$ from above:

$$
z_{\nu} \leq c
$$

Since $\Gamma$ satisfies the maximum principle, we conclude a bound for the $u_{\nu}$ from above. This concludes the proof of the proposition.

\section{HÖLDER A PRIORI ESTIMATES}

\subsection{Notation}

The Hölder estimates represent the core of the a priori estimates. We recall that for $\delta>0$

$$
C^{\delta, \frac{\delta}{2}}(Q)=\left\{\varphi|| \varphi\left(x_{1}, t_{1}\right)-\varphi\left(x_{2}, t_{2}\right) \mid \leq c\left(\left|x_{1}-x_{2}\right|^{\delta}+\left|t_{1}-t_{2}\right|^{\delta / 2}\right), \forall x_{1}, t_{1} ; x_{2}, t_{2} \in Q\right\} .
$$

We shall use the characterization of $C^{\delta, \frac{\delta}{2}}(Q)$ as a Campanato space. We need some notation. If $z_{0}=\left(x_{0}, t_{0}\right) \in Q$, we set

$$
\begin{aligned}
B_{R}\left(x_{0}\right) & =\left\{x|| x-x_{0} \mid \leq R\right\}, \\
\tilde{B}_{R}\left(x_{0}\right) & =B_{R}\left(x_{0}\right) \cap \Omega, \\
Q_{R}\left(z_{0}\right) & =B_{R}\left(x_{0}\right) \cap\left[\left(t_{0}-R^{2}\right)^{+}, t_{0}\right], \\
\tilde{Q}\left(z_{0}\right) & =\tilde{B}_{R}\left(x_{0}\right) \cap\left[\left(t_{0}-R^{2}\right)^{+}, t_{0}\right] .
\end{aligned}
$$

We shall write also $B_{R}, Q_{R}$ etc. when there is no ambiguity on the center.

$$
\varphi_{R, z_{0}}=\frac{\int_{\tilde{Q}_{R}\left(z_{0}\right)} \varphi \mathrm{d} x \mathrm{~d} t}{\left|\tilde{Q}_{R}\left(z_{0}\right)\right|},
$$


then the characterization of $C^{\delta, \frac{\delta}{2}}(Q)$ is given by (cf. Campanato [9], Da Prato [10])

$$
\int_{\tilde{Q}_{R}\left(z_{0}\right)}\left|\varphi-\varphi_{R, z_{0}}\right|^{2} \mathrm{~d} x \mathrm{~d} t \leq c R^{n+2+2 \delta}, \quad \forall z_{0} \in Q, R<R_{0}
$$

\subsection{Green function}

We shall make use of the Green functions associated to a point $z_{0} \in Q$ and a number $\theta>0$, denoted by $G_{x_{0}, t_{0}+\theta}(x, t)$, abbreviated as $G_{\theta}$, defined for $x \in R^{n}, t<t_{0}+\theta$, solution of

$$
\begin{aligned}
& \frac{\partial G_{\theta}}{\partial t}+\frac{\partial}{\partial x_{j}}\left(a_{i j} \frac{\partial G_{\theta}}{\partial x_{i}}\right)=0, \\
& G_{\theta}\left(x_{0}, t_{0}+\theta\right)=\delta\left(x-x_{0}\right) .
\end{aligned}
$$

This writing is formal, but $G_{\theta}$ is well defined for $t<t_{0}+\theta$, and satisfies the estimates (Aronson [1]):

$$
k_{1}\left(t_{0}+\theta-t\right)^{-\frac{n}{2}} \exp \left(-\delta_{1} \frac{\left|x-x_{0}\right|^{2}}{t_{0}+\theta-t}\right) \leq G_{\theta}(x, t) \leq k_{2}\left(t_{0}+\theta-t\right)^{-\frac{n}{2}} \exp \left(-\delta_{2} \frac{\left|x-x_{0}\right|^{2}}{t_{0}+\theta-t}\right)
$$

where $k_{1}, \delta_{1}, k_{2}, \delta_{2}$ are positive and depend only on $\alpha$ and the $L^{\infty}$ norm of the $a_{i j}$.

The function $s^{-\frac{n}{2}} \exp -\frac{\beta}{s}$ attains its maximum for $s>0$ at $\hat{s}=\frac{2 \beta}{n}$, and

$$
s^{-\frac{n}{2}} \exp -\frac{\beta}{s} \leq(\hat{s})^{-\frac{n}{2}}
$$

therefore we have, applying with $\beta=\delta_{2}\left|x-x_{0}\right|^{2}$,

$$
G_{\theta}(x, t) \leq c\left|x-x_{0}\right|^{-n}
$$

A sharper estimate can be obtained if $t_{0}+\theta-t$ is small compared to $\left|x-x_{0}\right|^{2}$. Indeed, if

$$
0<t_{0}+\theta-t<\varepsilon^{2}\left|x-x_{0}\right|^{2} \quad \text { with } \varepsilon^{2}<\frac{2 \delta_{2}}{n}
$$

then the above function is on its increasing side, and thus can be majorized by its value at $\varepsilon^{2}\left|x-x_{0}\right|^{2}$, hence

$$
G_{\theta}(x, t) \leq \delta(\varepsilon)\left|x-x_{0}\right|^{-n}
$$

with $\delta(\varepsilon)=k_{2} \varepsilon^{-n} \exp -\frac{\delta_{2}}{\varepsilon^{2}}$ tending to 0 as $\varepsilon \rightarrow 0$.

Furthermore, if

$$
\varepsilon^{2}\left|x-x_{0}\right|^{2} \leq t_{0}+\theta-t \leq m^{2}\left|x-x_{0}\right|^{2},
$$

we can write

$$
G_{\theta}(x, t) \geq \delta_{0}(\varepsilon)\left|x-x_{0}\right|^{-n}
$$

with $\delta_{0}(\varepsilon)=k_{1} m^{-n} \exp -\frac{\delta_{1}}{\varepsilon}$, tending to 0 as $\varepsilon \rightarrow 0$. 


\subsection{Basic inequalities}

We consider two cut off functions as follows:

$$
\tau(x)= \begin{cases}1 & \text { if }|x| \leq 1 \\ 0 & \text { if }|x| \geq 2\end{cases}
$$

$\tau \in C_{0}^{\infty}\left(R^{m}\right), 0 \leq \tau \leq 1$

$$
\beta(t)= \begin{cases}1 & \text { if } t \geq-1 \\ 0 & \text { if } t \leq-4\end{cases}
$$

$\beta \in C^{\infty}(R), 0 \leq \beta \leq 1$, and we set

$$
\begin{aligned}
\tau_{R, x_{0}} & =\tau_{R}=\tau\left(\frac{x-x_{0}}{R}\right), \\
\beta_{R, t_{0}} & =\beta_{R}=\beta\left(\frac{t-t_{0}}{R^{2}}\right), \\
\eta_{R, z_{0}}(x, t) & =\eta_{R}=\tau_{R, x_{0}} \beta_{R, t_{0}} .
\end{aligned}
$$

Note that $0 \leq \eta_{R} \leq 1, \eta_{R} \in C^{\infty}\left(R^{n+1}\right)$, and

$$
\eta_{R}= \begin{cases}1 & \text { if }(x, t) \in Q_{R}, \\ 0 & \text { if } x \notin B_{2 R} \text { or } t \leq t_{0}-4 R^{2} .\end{cases}
$$

We apply (3.15) as follows: we pick the constant $c$ in (3.2) such that

$$
c=c^{R}=0, \quad \text { if } B_{2 R}\left(x_{0}\right) \cap\left(R^{n}-\Omega\right) \neq \emptyset,
$$

and pick

$$
\psi= \begin{cases}\eta_{R}^{2} G_{\theta} & \text { if } t<t_{0} \\ 0 & \text { if } t>t_{0}\end{cases}
$$

Thanks to the choice of the constant (4.10) we can assert that

$$
\left(X_{1}-X_{1}(0)\right) \eta_{R}^{2}=0 \text { on } \Sigma .
$$

We write

$$
\begin{aligned}
\int_{Q}\left(\psi \frac{\partial X_{1}}{\partial t}+a_{i j} \frac{\partial X_{1}}{\partial x_{j}} \frac{\partial \psi}{\partial x_{i}}\right) & \mathrm{d} x \mathrm{~d} t \\
& =\int_{0}^{t_{0}} \int_{\Omega}\left(\eta_{R}^{2} G_{\theta} \frac{\partial}{\partial t}\left(X_{1}-X_{1}(0)\right)+a_{i j} \frac{\partial}{\partial x_{j}}\left(X_{1}-X_{1}(0)\right)\left(\frac{\partial G_{\theta}}{\partial x_{i}} \eta_{R}^{2}+G_{\theta} \frac{\partial \eta_{R}^{2}}{\partial x_{i}}\right)\right) \mathrm{d} x \mathrm{~d} t
\end{aligned}
$$


and using (4.3), we obtain

$$
\begin{aligned}
= & \int_{\Omega} \eta_{R}^{2} G_{\theta}\left(X_{1}-X_{1}(0)\right)\left(x, t_{0}\right) \mathrm{d} x-\int_{\Omega} \eta_{R}^{2} G_{\theta}\left(X_{1}-X_{1}(0)\right)(x, 0) \mathrm{d} x \\
& +\int_{0}^{t_{0}} \int_{\Omega} G_{\theta}\left(-\left(X_{1}-X_{1}(0)\right) \frac{\partial \eta_{R}^{2}}{\partial t}+a_{i j} \frac{\partial X_{1}}{\partial x_{j}} \frac{\partial}{\partial x_{i}} \eta_{R}^{2}\right) \mathrm{d} x \mathrm{~d} t \\
& -\int_{0}^{t_{0}} \int_{\Omega} \frac{\partial G_{\theta}}{\partial x_{i}} a_{i j} \frac{\partial}{\partial x_{j}} \eta_{R}^{2}\left(X_{1}-X_{1}(0)\right) \mathrm{d} x \mathrm{~d} t .
\end{aligned}
$$

Thus from (3.15) we deduce

$$
\begin{aligned}
& \alpha \int_{\tilde{Q}_{R}\left(z_{0}\right)}|D u|^{2} G_{\theta} \mathrm{d} x \mathrm{~d} t \leq \int_{\tilde{B}_{2 R}\left(x_{0}\right)} G_{\theta}\left(X_{1}-X_{1}(0)\right)(x, 0) \mathrm{d} x 1_{t_{0}<4 R^{2}} \\
& \quad+\int_{0}^{t_{0}} \int_{\Omega} G_{\theta}\left(\left(X_{1}-X_{1}(0)\right) \frac{\partial \eta_{R}^{2}}{\partial t}-a_{i j} \frac{\partial X_{1}}{\partial x_{j}} \frac{\partial}{\partial x_{i}} \eta_{R}^{2}\right) \mathrm{d} x \mathrm{~d} t \\
& +\int_{0}^{t_{0}} \int_{\Omega} \frac{\partial G_{\theta}}{\partial x_{i}} a_{i j} \frac{\partial}{\partial x_{j}} \eta_{R}^{2}\left(X_{1}-X_{1}(0)\right) \mathrm{d} x \mathrm{~d} t \\
& +c(\rho) \int_{\tilde{Q}_{2 R}}\left(G_{\theta} \sum_{y=1}^{N} k_{\nu}\right) \mathrm{d} x \mathrm{~d} t .
\end{aligned}
$$

Noting that, from the assumption on $k_{\nu}$

$$
\int_{\tilde{Q}_{2 R}}\left(G_{\theta} \sum_{\nu=1}^{N} k_{\nu}\right) \mathrm{d} x \mathrm{~d} t \leq c\left(\int_{Q_{2 R}} G_{\theta}^{q^{\prime}} \mathrm{d} x \mathrm{~d} t\right)^{\frac{1}{q^{\prime}}} \leq c R^{2-\frac{n+2}{q}} .
$$

Setting $\beta_{0}=2-\frac{n+2}{q}>0$, and making use of $(3.11,3.13)$ and the definition of the cut off functions, we obtain the basic inequality

Lemma 4.1. The following inequality holds:

$$
\begin{aligned}
\alpha \int_{\tilde{Q}_{R}\left(z_{0}\right)}|D u|^{2} G_{\theta} \mathrm{d} x \mathrm{~d} t \leq & c(\rho) \int_{\tilde{B}_{2 R}\left(x_{0}\right)}\left|u^{0}-c^{R}\right|^{2} G_{\theta}(x, 0) 1_{t_{0}<4 R^{2}} \\
& +c(\rho) \int_{\tilde{Q}_{2 R}\left(z_{0}\right)-\tilde{Q}_{R}\left(z_{0}\right)}\left(\frac{\left|u-c^{R}\right|^{2}}{R^{2}}+|D u|^{2}\right) G_{\theta} \mathrm{d} x \mathrm{~d} t \\
& +c(\rho) \int_{\left(t_{0}-4 R^{2}\right)^{+}}^{t_{0}} \int_{\tilde{B}_{2 R}-\tilde{B}_{R}} \eta_{R}^{2}\left|u-c^{R}\right|^{2}\left|D G_{\theta}\right|^{2} G_{\theta}^{-1} \mathrm{~d} x \mathrm{~d} t+c(\rho) R^{\beta_{0}} .
\end{aligned}
$$




\subsection{Auxiliary result}

To take care of the last integral in the right hand side of (4.14) one estimates the quantity

$$
Z=\int_{\left(t_{0}-4 R^{2}\right)^{+}}^{t_{0}} \int_{\tilde{B}_{2 R}-\tilde{B}_{R}} \eta_{R}^{2}\left|u-c^{R}\right|^{2}\left|D G_{\theta}\right|^{2} G_{\theta}^{-\frac{3}{2}} \mathrm{~d} x \mathrm{~d} t
$$

Let $\rho$ be a new cut off function such that

$$
\begin{aligned}
\rho \in C_{0}^{\infty}\left(\mathbb{R}^{n}\right), & \rho=0 \text { for }|x| \leq \frac{1}{2}, \\
0 \leq \rho \leq \tau, & \rho=\tau \text { for }|x| \geq 1,
\end{aligned}
$$

and set $\rho_{R}(x)=\rho\left(\frac{x-x_{0}}{R}\right)$ and

$$
\varphi_{R}(x, t)=\rho_{R}(x) \beta_{R}(t)
$$

so that

$$
\varphi_{R}=\eta_{R} \text { on }\left(\tilde{B}_{2 R}-\tilde{B}_{R}\right) \times\left[\left(t_{0}-4 R^{2}\right)^{+}, t_{0}\right] .
$$

We test the Green function equation (4.3) with $G_{\theta}^{-\frac{1}{2}}\left|u-c^{R}\right|^{2} \varphi_{R}^{2}$ and (2.22) with $\left(u-c^{R}\right) \varphi_{R}^{2} G_{\theta}^{\frac{1}{2}}$, integrating on $\left[\left(t_{0}-4 R^{2}\right)^{+}, t_{0}\right]$ and $\Omega$, and noting that these functions vanish on $\Sigma$. Combining we obtain

$$
\begin{aligned}
& \int_{\left(t_{0}-4 R^{2}\right)^{+}}^{t_{0}} \int_{\tilde{B}_{2 R}-\tilde{B}_{R / 2}} a_{i j} \frac{\partial G_{\theta}}{\partial x_{j}} \frac{\partial G_{\theta}}{\partial x_{i}} G_{\theta}^{-\frac{3}{2}}\left|u-c^{R}\right|^{2} \varphi_{R}^{2} \mathrm{~d} x \mathrm{~d} t \leq 4 \int_{\tilde{B}_{2 R}-\tilde{B}_{R / 2}} \rho_{R}^{2}\left|u^{0}-c^{R}\right|^{2} G_{\theta}^{\frac{1}{2}}(x, 0) \mathrm{d} x 1_{t_{0}<4 R^{2}} \\
& +8 \int_{\left(t_{0}-4 R^{2}\right)^{+}}^{t_{0}} \int_{\tilde{B}_{2 R}-\tilde{B}_{R / 2}} H\left(u-c^{R}\right) \varphi_{R}^{2} G_{\theta}^{\frac{1}{2}} \mathrm{~d} x \mathrm{~d} t+4 \int_{\left(t_{0}-4 R^{2}\right)^{+}}^{t_{0}} \int_{\tilde{B}_{2 R}-\tilde{B}_{R / 2}} a_{i j} \frac{\partial}{\partial x_{i}} G_{\theta}^{\frac{1}{2}} \frac{\partial}{\partial x_{j}} \varphi_{R}^{2}\left|u-c^{R}\right|^{2} \mathrm{~d} x \mathrm{~d} t \\
& +4 \int_{\left(t_{0}-4 R^{2}\right)+}^{t_{0}} \int_{\tilde{B}_{2 R}-\tilde{B}_{R / 2}} G_{\theta}^{\frac{1}{2}}\left[\left|u-c^{R}\right|^{2} \frac{\partial \varphi_{R}^{2}}{\partial t}-a_{i j} \frac{\partial}{\partial x_{j}}\left|u-c^{R}\right|^{2} \frac{\partial}{\partial x_{i}} \varphi_{R}^{2}\right] \mathrm{d} x \mathrm{~d} t .
\end{aligned}
$$

Majorizing the third integral on the right hand side by Young's inequality and combining with the left hand side, noting that $Z$ is smaller than the left hand side leads to

$$
Z \leq c \int_{\tilde{B}_{2 R}-\tilde{B}_{R / 2}}\left|u^{0}-c^{R}\right|^{2} G_{\theta}^{\frac{1}{2}}(x, 0) \mathrm{d} x 1_{t_{0}<4 R^{2}}+c \int_{\left(t_{0}-4 R^{2}\right)^{+}}^{t_{0}} \int_{\tilde{B}_{2 R}-\tilde{B}_{R / 2}}\left(\frac{\left|u-c^{R}\right|^{2}}{R^{2}}+|D u|^{2}\right) G_{\theta}^{\frac{1}{2}} \mathrm{~d} x \mathrm{~d} t+c(\rho) R^{\frac{n}{2}+\beta_{0}} .
$$




\subsection{Transformation of the basic inequality}

We want to make use of (4.18) to estimate the last integral in the right hand side of (4.14). We write it as the sum of two terms $(\varepsilon$ small $)$

$$
\begin{aligned}
I_{\varepsilon}= & \int_{\tilde{B}_{2 R}-\tilde{B}_{R}} \int_{\left(t_{0}-4 R^{2}\right)^{+}}^{t_{0} \wedge\left(t_{0}+\theta-\varepsilon^{2} R^{2}\right)^{+}} \eta_{R}^{2}\left|u-c^{R}\right|^{2}\left|D G_{\theta}\right|^{2} G_{\theta}^{-1} \mathrm{~d} x \mathrm{~d} t, \\
I I_{\varepsilon}= & \int_{\tilde{B}_{2 R}-\tilde{B}_{R}} \int_{t_{0} \wedge\left(t_{0}+\theta-\varepsilon^{2} R^{2}\right)^{+}}^{t_{0}} \eta_{R}^{2}\left|u-c^{R}\right|^{2}\left|D G_{\theta}\right|^{2} G_{\theta}^{-1} \mathrm{~d} x \mathrm{~d} t .
\end{aligned}
$$

Consider $I I_{\varepsilon}$. For $t_{0} \wedge\left(t_{0}+\theta-\varepsilon^{2} R^{2}\right)^{+}<t<t_{0}$, we have

$$
t_{0}+\theta-t<\varepsilon^{2} R^{2}<\varepsilon^{2}\left|x-x_{0}\right|^{2}
$$

since we integrate on $\tilde{B}_{2 R}-\tilde{B}_{R}$, hence $R<\left|x-x_{0}\right|<2 R$. Therefore from (4.7)

$$
G_{\theta}(x, t) \leq \delta(\varepsilon)\left|x-x_{0}\right|^{-n} \leq \delta(\varepsilon) R^{-n}
$$

hence, clearly

$$
I I_{\varepsilon} \leq \delta^{1 / 2}(\varepsilon) R^{-\frac{n}{2}} Z
$$

which implies from (4.18)

$$
\begin{aligned}
I I_{\varepsilon} \leq & c \int_{\tilde{B}_{2 R}-\tilde{B}_{R / 2}}\left|u^{0}-c^{R}\right|^{2}\left(G_{\theta}(x, 0)+\delta(\varepsilon) R^{-n}\right) \mathrm{d} x 1_{t_{0}<4 R^{2}} \\
& +c \int_{\left(t_{0}-4 R^{2}\right)^{+}} \int_{\tilde{B}_{2 R}-\tilde{B}_{R / 2}}\left(|D u|^{2}+\frac{\left|u-c^{R}\right|^{2}}{R^{2}}\right)\left(G_{\theta}+\delta(\varepsilon) R^{-n}\right) \mathrm{d} x \mathrm{~d} t+c(\rho) R^{\beta_{0}} .
\end{aligned}
$$

In $I_{\varepsilon}$, we use (4.5) and since

$$
R<\left|x-x_{0}\right|<2 R
$$

we have $G_{\theta} \leq c R^{-n}$, hence

$$
I_{\varepsilon} \leq c R^{-\frac{n}{2}} \int_{\tilde{B}_{2 R}-\tilde{B}_{R}} \int_{\left(t_{0}-4 R^{2}\right)^{+}}^{t_{0} \wedge\left(t_{0}+\theta-\varepsilon^{2} R^{2}\right)^{+}} \eta_{R}^{2}\left|u-c^{R}\right|^{2}\left|D G_{\theta}\right|^{2} G_{\theta}^{-3 / 2} \mathrm{~d} x \mathrm{~d} t .
$$


This integral is similar to $Z$ up to the upper level of integration of $t$. This leads to

$$
\begin{aligned}
I_{\varepsilon} \leq & c R^{-\frac{n}{2}} \int_{\tilde{B}_{2 R}-\tilde{B}_{R / 2}}\left|u^{0}-c^{R}\right|^{2} G_{\theta}^{\frac{1}{2}}(x, 0) \mathrm{d} x 1_{t_{0}<4 R^{2}} \\
& +c R^{-\frac{n}{2}} \int_{\left(t_{0}-4 R^{2}\right)^{+}}^{t_{0} \wedge\left(t_{0}+\theta-\varepsilon^{2} R^{2}\right)^{+}} \int_{\tilde{B}_{2 R}-\tilde{B}_{R / 2}}\left(\frac{\left|u-c^{R}\right|^{2}}{R^{2}}+|D u|^{2}\right) G_{\theta}^{\frac{1}{2}} \mathrm{~d} x \mathrm{~d} t+c(\rho) R^{\beta_{0}} .
\end{aligned}
$$

For

$$
\left(t_{0}-4 R^{2}\right)^{+}<t<t_{0} \wedge\left(t_{0}+\theta-\varepsilon^{2} R^{2}\right)^{+}
$$

we have, since $x \in \tilde{B}_{2 R}-\tilde{B}_{R / 2}$

$$
\frac{\varepsilon^{2}}{4}\left|x-x_{0}\right|^{2}<\varepsilon^{2} R^{2}<t_{0}+\theta-t<4 R^{2}+\theta .
$$

Suppose we restrict $\theta$ so that

$$
\theta \leq q^{2} R^{2}, \quad q>1
$$

then

$$
\frac{\varepsilon^{2}}{4}\left|x-x_{0}\right|^{2}<t_{0}+\theta-t<\left(4+q^{2}\right) R^{2}<4\left(4+q^{2}\right)\left|x-x_{0}\right|^{2}
$$

and thus (4.8) applies with $\varepsilon$ changed into $\frac{\varepsilon}{2}$ and $m^{2}=4\left(4+q^{2}\right)$.

It follows that

$$
G_{\theta}(x, t) \geq \delta\left(\frac{\varepsilon}{2}\right)\left|x-x_{0}\right|^{-n} \geq \delta_{0}\left(\frac{\varepsilon}{2}\right) 2^{-n} R^{-n} .
$$

Using

$$
G_{\theta}(x, 0) \leq c R^{-n}
$$

we finally obtain

$$
I_{\varepsilon} \leq c R^{-n} \int_{\tilde{B}_{2 R}}\left|u^{0}-c^{R}\right|^{2} \mathrm{~d} x 1_{t_{0}<4 R^{2}}+c K(\varepsilon) \int_{\left(t_{0}-4 R^{2}\right)^{+}}^{t_{0} \wedge\left(t_{0}+\theta-\varepsilon^{2} R^{2}\right)^{+}} \int_{\tilde{B}_{2 R}-\tilde{B}_{R / 2}}\left(\frac{\left|u-c^{R}\right|^{2}}{R^{2}}+|D u|^{2}\right) G_{\theta} \mathrm{d} x \mathrm{~d} t+c(\rho) R^{\beta_{0}}
$$

with $K(\varepsilon)=2^{\frac{n}{2}} \delta_{0}^{-\frac{1}{2}}\left(\frac{\varepsilon}{2}\right) \rightarrow+\infty$ as $\varepsilon \rightarrow 0$. 
Collecting results we transform the basic inequality (4.14) as follows:

Lemma 4.2. The following inequality holds (provided (4.20) holds)

$$
\begin{aligned}
\alpha \int_{\tilde{Q}_{R}\left(z_{0}\right)}|D u|^{2} G_{\theta} \mathrm{d} x \mathrm{~d} t \leq & c(\rho) \int_{B_{2 R}\left(x_{0}\right)}\left|u^{0}-c^{R}\right|^{2}\left(G_{\theta}(x, 0)+R^{-n}\right) 1_{t_{0}<4 R^{2}} \\
& +c(\rho) \int_{\tilde{Q}_{2 R}\left(z_{0}\right)-\tilde{Q}_{R / 2}\left(z_{0}\right)}|D u|^{2}\left(K(\varepsilon) G_{\theta}+\delta(\varepsilon) R^{-n}\right) \mathrm{d} x \mathrm{~d} t \\
& +c(\rho) \int_{\tilde{Q}_{2 R}\left(z_{0}\right)-\tilde{Q}_{R / 2}\left(z_{0}\right)} \frac{\left|u-c^{R}\right|^{2}}{R^{2}}\left(G_{\theta}+\delta(\varepsilon) R^{-n}\right) \mathrm{d} x \mathrm{~d} t \\
& +c(\rho) K(\varepsilon) \int_{t_{0} \wedge\left(t_{0}+\theta-\varepsilon^{2} R^{2}\right)^{+}} \int_{\left(t_{0}-4 R^{2}\right)^{+}} \frac{\left|u-c^{R}\right|^{2}}{R^{2}} G_{\theta} \mathrm{d} x \mathrm{~d} t+c(\rho) R^{\beta_{0} / 2}
\end{aligned}
$$

with $\delta(\varepsilon) \rightarrow 0, K(\varepsilon) \rightarrow \infty$ as $\varepsilon \rightarrow 0$.

\subsection{Choice of the constant $c^{R}$}

In the case $(4.10)$ we have $c^{R}=0$. Since $u^{0}$ vanishes on the boundary, we have

$$
\left|u^{0}(x)\right| \leq c R, \quad \text { for } x \in \tilde{B}_{2 R}\left(x_{0}\right)
$$

and thus

$$
\int_{\tilde{B}_{2 R}\left(x_{0}\right)}\left|u^{0}\right|^{2}\left(G_{\theta}(x, 0)+R^{-n}\right) \mathrm{d} x \leq c R^{2} .
$$

Next, considering the integral

$$
\int_{\left(t_{0}-4 R^{2}\right)^{+}}^{t_{0} \wedge\left(t_{0}+\theta-\varepsilon^{2} R^{2}\right)^{+}} \int_{\tilde{B}_{2 R}-\tilde{B}_{R / 2}} \frac{|u|^{2}}{R^{2}} G_{\theta} \mathrm{d} x \mathrm{~d} t,
$$

we may use inside the integral

$$
G_{\theta} \leq c R^{-n} \leq K(\varepsilon) G_{\theta}
$$

together with Poincaré's inequality to assert that

$$
I_{1} \leq c K(\varepsilon) \int_{\tilde{Q}_{4 R}-\tilde{Q}_{R / 2}}|D u|^{2} G_{\theta} \mathrm{d} x \mathrm{~d} t .
$$


Next

$$
I_{2}=\int_{\tilde{Q}_{2 R}-\tilde{Q}_{R / 2}} \frac{|u|^{2}}{R^{2}} G_{\theta} \mathrm{d} x \mathrm{~d} t=\int_{\tilde{B}_{2 R}-\tilde{B}_{R / 2}} \int_{\left(t_{0}-4 R^{2}\right)^{+}}^{t_{0}} \frac{|u|^{2}}{R^{2}} G_{\theta} \mathrm{d} x \mathrm{~d} t+\int_{\tilde{B}_{R / 2}\left(t_{0}-4 R^{2}\right)^{+}} \int_{\left.t^{2}-\frac{R^{2}}{4}\right)^{+}}^{\frac{|u|^{2}}{R^{2}}} G_{\theta} \mathrm{d} x \mathrm{~d} t .
$$

In the second integral, we may use inside

$$
c R^{-n} \leq G_{\theta} \leq c R^{-n}
$$

together with Poincaré's inequality. In the first integral we decompose the interval of time into $\left[\left(t_{0}-4 R^{2}\right)^{+}\right.$, $\left.t_{0} \wedge\left(t_{0}+\theta-\varepsilon^{2} R^{2}\right)^{+}\right]$and $\left[t_{0} \wedge\left(t_{0}+\theta-\varepsilon^{2} R^{2}\right)^{+}, t_{0}\right]$. In the first interval we recover $I_{1}$. In the second interval we can majorize $G_{\theta}$ by $\delta(\varepsilon) R^{-n}$, and use Poincaré again.

Collecting results we can assert that

$$
\alpha \int_{\tilde{Q}_{R}\left(z_{0}\right)}|D u|^{2} G_{\theta} \mathrm{d} x \mathrm{~d} t \leq \int_{\tilde{Q}_{4 R}\left(z_{0}\right)-\tilde{Q}_{R / 2}\left(z_{0}\right)}|D u|^{2}\left(G_{\theta} K_{1}(\varepsilon)+\delta_{1}(\varepsilon) R^{-n}\right) \mathrm{d} x \mathrm{~d} t+c(\rho) R^{\beta_{0}},
$$

where again

$$
\delta_{1}(\varepsilon) \rightarrow 0, \quad K_{1}(\varepsilon) \rightarrow \infty \quad \text { as } \varepsilon \rightarrow 0 .
$$

We now assume that

$$
B_{2 R}\left(x_{0}\right) \subset \Omega
$$

Note that $\tilde{B}_{2 R}=B_{2 R}, \tilde{Q}_{2 R}=Q_{2 R}$.

To proceed, we introduce the notation

$$
u_{R, x_{0}, t}^{\rho}=\frac{\int_{B_{2 R}} u(x, t) \rho_{R} \mathrm{~d} x}{\int_{B_{2 R}} \rho_{R} \mathrm{~d} x},
$$

where $\rho_{R}$ has been defined in (4.16). We shall need the following result:

Lemma 4.3. Let $\left(t_{0}-4 R^{2}\right)^{+} \leq s \leq t \leq t_{0}$, then one has

$$
\left|u_{R, x_{0}, t}^{\rho}-u_{R, x_{0}, s}^{\rho}\right|^{2} \leq c R^{-n} \int_{s}^{t} \int_{B_{2 R}-B_{R / 2}}|D u|^{2}+c R^{2 \beta_{0}} .
$$

Proof. It is obtained by testing (2.22) with $\rho_{R}$ and integrating over $x$ and the interval $s, t$.

Consider the case

$$
t_{0}+\theta-\varepsilon^{2} R^{2} \leq 0
$$

then we take

$$
c^{R}=\left(u^{0}\right)^{\rho}=u_{R, x_{0}, 0}^{\rho}
$$


Note that in $B_{2 R}\left(x_{0}\right)$, we have $\left|u(x)-\left(u^{0}\right)^{\rho}\right| \leq c R$, hence the first term in the right hand side of (4.22) is bounded by $c(\rho) R^{2}$. Next applying (4.26) we have

$$
\left|u_{R, x_{0}, t}^{\rho}-c^{R}\right|^{2} \leq c R^{-n} \int_{0}^{t_{0}} \int_{B_{2 R}-B_{R / 2}}|D u|^{2}+c R^{2 \beta_{0}} .
$$

Moreover, in (4.22) the last integral vanishes. Consider

$$
\begin{aligned}
\int_{Q_{2 R}-Q_{R / 2}} \frac{\left|u-c^{R}\right|^{2}}{R^{2}}\left(G_{\theta}+\delta(\varepsilon) R^{-n}\right) \mathrm{d} x \mathrm{~d} t & =\int_{0}^{t_{0}} \int_{B_{2 R}-B_{R / 2}} \frac{\left|u-c^{R}\right|^{2}}{R^{2}}\left(G_{\theta}+\delta(\varepsilon) R^{-n}\right) \mathrm{d} x \mathrm{~d} t \\
& \leq \delta^{\prime}(\varepsilon) R^{-n} \int_{0}^{t_{0}} \int_{B_{2 R}-B_{R / 2}} \frac{\left|u-c^{R}\right|^{2}}{R^{2}} \mathrm{~d} x \mathrm{~d} t
\end{aligned}
$$

since in the integral $G_{\theta}(x, t) \leq 2^{-n} \delta(2 \varepsilon) R^{-n}$, by $(4.7)$.

Using

$$
\int_{0}^{t_{0}} \int_{B_{2 R}-B_{R / 2}} \frac{\left|u-c^{R}\right|^{2}}{R^{2}} \mathrm{~d} x \mathrm{~d} t \leq 2 \int_{0}^{t_{0}} \int_{B_{2 R}-B_{R / 2}} \frac{\left|u-u_{R, x_{0}, t}^{\rho}\right|^{2}}{R^{2}} \mathrm{~d} x \mathrm{~d} t+2 R^{n-2} \int_{0}^{t_{0}}\left|u_{R, x_{0}, t}^{\rho}-c^{R}\right|^{2} \mathrm{~d} t
$$

and Poincaré's inequality, we obtain again that the inequality (4.24) holds.

It remains to consider the case

$$
t_{0}+\theta-\varepsilon^{2} R^{2}>0 .
$$

We take this time

$$
c^{R}=\frac{\int_{\left(t_{0}-4 R^{2}\right)^{+}}^{t_{0} \wedge\left(t_{0}+\theta-\varepsilon^{2} R^{2}\right)} u_{R, x_{0}, t}^{\rho} \mathrm{d} t}{\int_{\left(t_{0}-4 R^{2}\right)^{+}}^{t_{0} \wedge\left(t_{0}+\theta-\varepsilon^{2} R^{2}\right)} \mathrm{d} t} .
$$

To evaluate the first term on the right hand side of (4.22), we restrict ourselves to $t_{0}<4 R^{2}$. We remark that

$$
\left|c^{R}-\left(u^{0}\right)^{\rho}\right|^{2} \leq \frac{\int_{0}^{t_{0} \wedge\left(t_{0}+\theta-\varepsilon^{2} R^{2}\right)}\left|u_{R, x_{0}, t}^{\rho}-\left(u^{0}\right)^{\rho}\right|^{2} \mathrm{~d} t}{\int_{0}^{t_{0} \wedge\left(t_{0}+\theta-\varepsilon^{2} R^{2}\right)} \mathrm{d} t},
$$

and from Lemma 4.3

$$
\left|c^{R}-\left(u^{0}\right)^{\rho}\right|^{2} \leq c R^{-n} \int_{0}^{t_{0} \wedge\left(t_{0}+\theta-\varepsilon^{2} R\right)} \int_{B_{2 R}-B_{R / 2}}|D u|^{2} \mathrm{~d} x \mathrm{~d} t+c R^{2 \beta_{0}} .
$$


Using inside the integral $G_{\theta}(x, t) \geq \delta_{0}\left(\frac{\varepsilon}{2}\right) 2^{-n} R^{-n}$, we obtain

$$
\left|c^{R}-\left(u^{0}\right)^{\rho}\right|^{2} \leq K^{\prime}(\varepsilon) \underset{Q_{2 R}\left(z_{0}\right)-Q_{R / 2}\left(z_{0}\right)}{ }|D u|^{2} G_{\theta}(x, t) \mathrm{d} x \mathrm{~d} t+c R^{2 \beta_{0}},
$$

which is similar to the right hand side of (4.24).

Consider the term

$$
J=\int_{Q_{2 R}\left(z_{0}\right)-Q_{R / 2}\left(z_{0}\right)} \frac{\left|u-c^{R}\right|^{2}}{R^{2}}\left(G_{\theta}+\delta(\varepsilon) R^{-n}\right) \mathrm{d} x \mathrm{~d} t,
$$

then we can reduce it to the study of

$$
J_{1}=\int_{Q_{2 R}\left(z_{0}\right)-Q_{R / 2}\left(z_{0}\right)} \frac{\left|u-u_{R, x_{0}, t}^{\rho}\right|^{2}}{R^{2}}\left(G_{\theta}+\delta(\varepsilon) R^{-n}\right) \mathrm{d} x \mathrm{~d} t
$$

and

$$
J_{2}=\int_{Q_{2 R}\left(z_{0}\right)-Q_{R / 2}\left(z_{0}\right)} \frac{\left|u_{R, x_{0}, t}^{\rho}-c^{R}\right|^{2}}{R^{2}}\left(G_{\theta}+\delta(\varepsilon) R^{-n}\right) \mathrm{d} x \mathrm{~d} t .
$$

We treat $J_{1}$ as in the case when $c^{R}=0$ (see above). Note that

$$
J_{2} \leq c \int_{\left(t_{0}-4 R^{2}\right)^{+}}^{t_{0}} \frac{\left|u_{R, x_{0}, t}^{\rho}-c^{R}\right|^{2}}{R^{2}} \mathrm{~d} t
$$

and the interval of integration in time is split into

$$
\left[\left(t_{0}-4 R^{2}\right)^{+}, t_{0} \wedge\left(t_{0}+\theta-\varepsilon^{2} R^{2}\right)\right] \quad \text { and } \quad\left[t_{0} \wedge\left(t_{0}+\theta-\varepsilon^{2} R^{2}\right), t_{0}\right]
$$

In the second interval, we use the fact that

$$
\int_{t_{0} \wedge\left(t_{0}+\theta-\varepsilon^{2} R^{2}\right)}^{t_{0}} \frac{\left|u_{R, x_{0}, t}^{\rho}-u_{R, x_{0}, t_{0} \wedge\left(t_{0}+\theta-\varepsilon^{2} R^{2}\right)}^{\rho}\right|^{2}}{R^{2}} \mathrm{~d} t \leq c \varepsilon^{2}\left[R^{-n} \int_{\left(t_{0}-4 R^{2}\right)+B_{2 R}-B_{R / 2}}^{t_{0}}|D u|^{2} \mathrm{~d} x \mathrm{~d} t+R^{2 \beta_{0}}\right],
$$

which is a term like in the right hand side of (4.24).

For $\left|u_{R, x_{0}, t_{0} \wedge\left(t_{0}+\theta-\varepsilon^{2} R^{2}\right)}^{\rho}-c^{R}\right|^{2}$ and the first interval of time we need to use (4.26) with $s \leq t \leq t_{0} \wedge\left(t_{0}+\right.$ $\theta-\varepsilon^{2} R^{2}$ ), in which case we rely on the fact that $R^{-n} \leq K(\varepsilon) G_{\theta}(x, t)$ to obtain contributions of the type of the right hand side of (4.24).

Collecting results, thanks to the appropriate choice of $c^{R}$, we can assert that:

Lemma 4.4. Provided (4.20) holds, the inequality (4.24) applies, with $\delta_{1}(\varepsilon) \rightarrow 0, K_{1}(\varepsilon) \rightarrow \infty$ as $\varepsilon \rightarrow 0$. 


\subsection{Statement of the main property}

We can now state the

Proposition 4.1. Under the conditions of Proposition 3.1 one has the property

$$
\int_{\tilde{Q}_{R\left(z_{0}\right)}}|D u|^{2} \mathrm{~d} x \mathrm{~d} t \leq c(\rho) R^{n+2 \beta}
$$

for some $\beta>0,2 \beta<\beta_{0}$.

Proof. Using

$$
c R^{-n} \leq G_{R^{2}}(x, t) \quad \text { for } \quad t<t_{0}, x \in B_{4 R}\left(x_{0}\right)
$$

we assert from (4.24), that for $\theta<q^{2} R^{2}$,

$$
\int_{\tilde{Q}_{\frac{R}{2}}\left(z_{0}\right)}|D u|^{2} G_{\theta} \mathrm{d} x \mathrm{~d} t \leq K(\varepsilon) \int_{\tilde{Q}_{4 R\left(z_{0}\right)}-\tilde{Q}_{\frac{R}{2}\left(z_{0}\right)}}|D u|^{2} G_{\theta} \mathrm{d} x \mathrm{~d} t+\delta(\varepsilon) \int_{\tilde{Q}_{4 R\left(z_{0}\right)}-\tilde{Q}_{\frac{R}{2}\left(z_{0}\right)}} G_{R^{2}} \mathrm{~d} x \mathrm{~d} t+c(\rho) R^{\beta_{0}}
$$

Then for $2 \beta<\beta_{0}$ and $8^{2 \beta}<1$, setting

$$
\varphi(R)=R^{-2 \beta} \sup _{\theta<q^{2} R^{2}} \int_{\tilde{Q}_{R / 2}}|D u|^{2} G_{\theta} \mathrm{d} x \mathrm{~d} t
$$

we deduce, by the hole filling trick that

$$
\varphi(R) \leq \nu(\varepsilon) \varphi(8 R)+c(\rho)
$$

where $\nu(\varepsilon)<1$ for a convenient choice of $\varepsilon$ sufficiently small. This implies

$$
\sup _{0<R<R_{1}} R^{-2 \beta} \sup _{\theta<4 q^{2} R^{2}} \int_{\tilde{Q}_{R}}|D u|^{2} G_{\theta} \mathrm{d} x \mathrm{~d} t \leq c(\rho),
$$

and in particular

$$
R^{-2 \beta} \int_{\tilde{Q}_{R}}|D u|^{2} G_{R^{2}} \mathrm{~d} x \mathrm{~d} t \leq c(\rho)
$$

hence also (4.32), and the proof has been completed.

To obtain the Hölder property from (4.32), it is sufficient to observe that

$$
\int_{\tilde{Q}_{R}\left(z_{0}\right)}\left|u-u_{R, z_{0}}\right|^{2} \mathrm{~d} x \mathrm{~d} t \leq c R^{2} \int_{\tilde{Q}_{4 R\left(z_{0}\right)}}|D u|^{2} \mathrm{~d} x \mathrm{~d} t+c R^{n+2+2 \beta_{0}} .
$$

When $B_{2 R}\left(x_{0}\right) \cap\left(R^{n}-\Omega\right) \neq \emptyset$, this follows from Poincaré's inequality. When $B_{2 R}\left(x_{0}\right) \subset \Omega$, it is obtained by using the analogue of Lemma 4.2 with $\tau_{R}$ as a test function. 
From (4.32) and (4.33), thanks to the characterization (4.2), we obtain the Hölder estimate

$$
\int_{\tilde{Q}_{R}\left(z_{0}\right)}\left|u-u_{R, z_{0}}\right|^{2} \mathrm{~d} x \mathrm{~d} t=c(\rho) R^{n+2+2 \beta} .
$$

Remark 4.1. Note that $\beta$ does not depend on $\rho$, but only on $\beta_{0}=2-\frac{n+2}{q}$.

\section{A PRIORI ESTimates In $L^{p}\left(0, T ; W^{2, p}(\Omega)\right)$}

\subsection{Statement of the result}

We want to show now an estimate of $u$ in $L^{p}\left(0, T ; W^{2, p}\right)$ and $\frac{\partial u}{\partial t} \in L^{p}(Q)$, with $p \leq q$.

We shall rely on the method used in the elliptic theory (see Frehse [11]). We need to know that $u$ is Hölder and the following property of the Hamiltonian summarizes what is needed

$$
|H(x, t, u, D u)| \leq K|D u|^{2}+f, \quad f \in L^{q}>\frac{n}{2}+1,
$$

which of course is satisfied in the conditions of Proposition 3.1. Without loss of generality we can take

$$
u^{0}=0
$$

Indeed defining $z$ by

$$
\begin{aligned}
\frac{\partial z}{\partial t}+A(t) z & =0, \\
\left.z\right|_{\Sigma} & =0,
\end{aligned}
$$

then from the linear theory and the assumption (2.20), $z$ has full regularity. By a simple translation, we obtain a problem where the property (5.1) is satisfied as well as (5.2).

We state the

Proposition 5.1. Under the conditions of Proposition 3.1 one has the property

$$
\left\|\frac{\partial u}{\partial t}\right\|_{L^{p}(Q)}+\|u\|_{L^{p}\left(0, T ; W^{2, p}(\Omega)\right)} \leq c(\rho) \quad \forall p \leq q .
$$

\subsection{Proof of Proposition 5.1}

Let $Q_{R}\left(x_{0}\right)$ be the cube of center $x_{0}$ and side length $R$. We note

$$
\tilde{Q}_{R}\left(x_{0}\right)=\Omega \cap Q_{R}\left(x_{0}\right) .
$$

Let $\tau$ be a Lipschitz continuous function such that

$$
\tau=\left\{\begin{array}{lll}
1 & \text { on } & Q_{1}(0), \\
0 & \text { outside } & Q_{2}(0),
\end{array}\right.
$$

and denote

$$
\tau_{R}(x)=\tau\left(\frac{x-x_{0}}{R}\right)=\left\{\begin{array}{lll}
1 & \text { on } & Q_{R}\left(x_{0}\right) \\
0 & \text { outside } & Q_{2 R}\left(x_{0}\right)
\end{array}\right.
$$


We shall denote by $K$ constants depending only on the data, $K(\rho)$ those depending also on the $L^{\infty}$ norm of $u$, which has been denoted by $\rho$, and $K_{R}$ constants depending on $R$. Calculating

$$
\frac{\partial}{\partial t}\left(u^{\nu} \tau_{R}\right)+A(t)\left(u^{\nu} \tau_{R}\right)
$$

and applying the linear theory of parabolic equations, we can assert, making use of (5.1):

$$
\left(\int_{0}^{T} \int_{\tilde{Q}_{R}}\left|\frac{\partial u}{\partial t}\right|^{p} \mathrm{~d} x \mathrm{~d} t\right)^{\frac{1}{p}}+\left(\int_{0}^{T} \int_{\tilde{Q}_{R}}\left|D^{2} u\right|^{p} \mathrm{~d} x \mathrm{~d} t\right)^{\frac{1}{p}} \leq K\left(\int_{0}^{T} \int_{\tilde{Q}_{2 R}}|D u|^{2 p} \mathrm{~d} x \mathrm{~d} t\right)^{\frac{1}{p}}+K_{R}, \quad \forall p \leq q
$$

We notice that

$$
\int_{0}^{T} \int_{\tilde{Q}_{2 R}}|D u|^{2 p} \mathrm{~d} x \mathrm{~d} t \leq \int_{0}^{T} \int_{\tilde{Q}_{4 R}}\left|\tau_{2 R} D u\right|^{2 p} \mathrm{~d} x \mathrm{~d} t .
$$

Let $c^{R}(t)$ be a vector, depending on $t$ but not on $x$, such that

$$
\begin{aligned}
c^{R} & =0 \quad \text { if } \quad Q_{4 R}\left(x_{0}\right) \cap\left(\mathbb{R}^{n}-\Omega\right) \neq \emptyset \\
c^{R}(t) & =\frac{1}{2}\left(\min _{x \in Q_{4 R}} u(x, t)+\max _{x \in Q_{4 R}} u(x, t)\right) \text { if } Q_{4 R}\left(x_{0}\right) \subset \Omega .
\end{aligned}
$$

Therefore $\tau_{2 R}\left(u-c^{R}\right)=0$ on $\Sigma$.

Performing an integration by parts we have

$$
\begin{aligned}
\int_{0}^{T} \int_{\tilde{Q}_{4 R}}\left|\tau_{2 R} D u\right|^{2 p} \mathrm{~d} x \mathrm{~d} t= & -\int_{0}^{T} \int_{\tilde{Q}_{4 R}}^{T} \tau_{2 R}^{2 p}|D u|^{2 p-2} \Delta u_{\nu}\left(u_{\nu}-c_{\nu}^{R}\right) \mathrm{d} x \mathrm{~d} t \\
& -2 p \int_{0}^{T} \int_{\tilde{Q}_{4 R}} \tau_{2 R}^{2 p-1} \frac{\partial \tau_{2 R}}{\partial x_{i}}|D u|^{2 p-2} \frac{\partial u_{\nu}}{\partial x_{i}}\left(u_{\nu}-c_{\nu}^{R}\right) \mathrm{d} x \mathrm{~d} t \\
& -2(p-1) \int_{0}^{T} \int_{\tilde{Q}_{4 R}} \tau_{2 R}^{2 p}|D u|^{2 p-4} \frac{\partial u_{\mu}^{2}}{\partial x_{i} \partial x_{j}} \frac{\partial u_{\mu}}{\partial x_{j}} \frac{\partial u_{\nu}}{\partial x_{i}}\left(u_{\nu}-c_{\nu}^{R}\right) \mathrm{d} x \mathrm{~d} t \\
\leq & c_{p} \int_{0}^{T} \int_{\tilde{Q}_{4 R}}^{T} \tau_{2 R}^{2 p}|D u|^{2 p-2}\left|D^{2} u\right|\left|u-c^{R}\right| \mathrm{d} x \mathrm{~d} t \\
& +c_{p} \int_{0}^{T} \int_{\tilde{Q}_{4 R}} \tau_{2 R}^{2 p-1}\left|D \tau_{2 R}\right||D u|^{2 p-1}\left|u-c^{R}\right| \mathrm{d} x \mathrm{~d} t
\end{aligned}
$$


hence

$$
\leq c_{p} \int_{0}^{T} \int_{\tilde{Q}_{4 R}} \tau_{2 R}^{2 p}\left|D^{2} u\right|^{p}\left|u-c^{R}\right| \mathrm{d} x \mathrm{~d} t+c_{p} \int_{0}^{T} \int_{\tilde{Q}_{4 R}} \tau_{2 R}^{2 p}|D u|^{2 p}\left|u-c^{R}\right| \mathrm{d} x \mathrm{~d} t+c_{p} \int_{0}^{T} \int_{\tilde{Q}_{4 R}}\left|D \tau_{2 R}\right|^{2 p}\left|u-c^{R}\right| \mathrm{d} x \mathrm{~d} t,
$$

and from the Hölderianity of $u$

$$
\leq K_{p}(\rho) R^{\beta} \int_{0}^{T} \int_{\tilde{Q}_{4 R}} \tau_{2 R}^{2 p}|D u|^{2 p} \mathrm{~d} x \mathrm{~d} t+K_{p}(\rho) R^{\beta} \int_{0}^{T} \int_{\tilde{Q}_{4 R}} \tau_{2 R}^{2 p}\left|D^{2} u\right|^{p} \mathrm{~d} x \mathrm{~d} t+K_{p, R}(\rho) .
$$

Combining with $(5.4,5.5)$, we arrive at

$$
\int_{0}^{T} \int_{\tilde{Q}_{R}}\left|D^{2} u\right|^{p} \mathrm{~d} x \mathrm{~d} t \leq \frac{K_{p}^{\prime}(\rho) R^{\beta}}{1-K_{p}(\rho) R^{\beta}} \int_{0}^{T} \int_{\tilde{Q}_{4 R}}\left|D^{2} u\right|^{p} \mathrm{~d} x \mathrm{~d} t+K_{p, R}^{\prime}(\rho)
$$

provided of course $1-K_{p}(\rho) R^{\beta}>0$. So take $R<R_{1}$ with $1-K_{p}(\rho) R_{1}^{\beta}>0$ and define

$$
\xi=\sup _{\substack{x_{0} \in \Omega \\ R<R_{1}}} \int_{0}^{T} \int_{\tilde{Q}_{R}}\left|D^{2} u\right|^{p} \mathrm{~d} x \mathrm{~d} t
$$

then notice that

$$
\sup _{\substack{x_{0} \in \Omega \\ R<R_{1}}} \int_{0}^{T} \int_{\tilde{Q}_{4 R}}\left|D^{2} u\right|^{p} \mathrm{~d} x \mathrm{~d} t \leq K \xi
$$

and thus

$$
\xi \leq \frac{K_{p}^{\prime \prime}(\rho) R^{\beta}}{1-K_{p}(\rho) R^{\beta}} \xi+K_{p, R}^{\prime}(\rho)
$$

We may assume $R_{1}$ such that

$$
\frac{K_{p}^{\prime \prime}(\rho) R_{1}^{\beta}}{1-K_{p}(\rho) R_{1}^{\beta}}<1,
$$

which leads to an estimate on $\xi$. By a finite covering of $\Omega$ by cubes $Q_{R}$, we easily conclude the estimate (5.3). The proof has been completed.

\section{Proof of Theorem 2.1}

If we consider the approximated Hamiltonians

$$
H^{\varepsilon}(x, t, \lambda, p)=\frac{H(x, t, \lambda p)}{1+\varepsilon|H(x, t, \lambda, p)|},
$$


then it is clear that it satisfies all the assumptions of $H$, with the same constants, independently of $\varepsilon$. Now if we consider the approximate problem

$$
\begin{array}{r}
\frac{\partial u^{\varepsilon}}{\partial t}+A(t) u^{\varepsilon}=H^{\varepsilon}\left(x, t, u^{\varepsilon}, D u^{\varepsilon}\right), \\
u^{\varepsilon}(0)=u^{0},\left.\quad u^{\varepsilon}\right|_{\varepsilon}=0,
\end{array}
$$

whose solution in $L^{2}\left(0, T ; W^{2, p}(\Omega)\right), \frac{\partial u^{\varepsilon}}{\partial t} \in L^{p}(Q)$, exists for any $p$, since $H^{\varepsilon}$ is bounded by $\frac{1}{\varepsilon}$, then the a priori estimates obtained in $(3.18,3.19,4.34)$ and $(5.3)$ are valid for $u^{\varepsilon}$. By extracting a subsequence which converges in the appropriate spaces, it is fairly easy to see that the limit is a solution of $(2.21-2.23)$.

\section{REFERENCES}

[1] D.G. Aronson, Bounds for Fundamental Solution of a Parabolic Equation. Bull. Amer. Math. Soc. 73 (1968) 890-896.

[2] A. Bensoussan and J. Frehse, Regularity of Solutions of Systems of Partial Differential Equations and Applications. Springer Verlag (to be published).

[3] A. Bensoussan and J. Frehse, Nonlinear elliptic systems in stochastic game theory. J. Reine Angew. Math. 350 (1984) $23-67$.

[4] A. Bensoussan and J. Frehse, $C^{\alpha}$-Regularity Results for Quasi-Linear Parabolic Systems. Comment. Math. Univ. Carolin. 31 (1990) 453-474.

[5] A. Bensoussan and J. Frehse, Ergodic Bellman systems for stochastic games, in Differential equations, dynamical systems, and control science. Dekker, New York (1994) 411-421.

[6] A. Bensoussan and J. Frehse, Ergodic Bellman systems for stochastic games in arbitrary dimension. Proc. Roy. Soc. London Ser. A 449 (1935) 65-77.

[7] A. Bensoussan and J. Frehse, Stochastic games for $N$ players. J. Optim. Theory Appl. 105 (2000) 543-565. Special Issue in honor of Professor David G. Luenberger.

[8] A. Bensoussan and J.-L. Lions, Impulse control and quasivariational inequalities. Gauthier-Villars (1984). Translated from the French by J.M. Cole.

[9] S. Campanato, Equazioni paraboliche del secondo ordine e spazi $L^{2, \theta}(\Omega, \delta)$. Ann. Mat. Pura Appl. (4) 73 (1966) 55-102.

[10] G. Da Prato, Spazi $L^{(p, \theta)}(\Omega, \delta)$ e loro proprietà. Ann. Mat. Pura Appl. (4) 69 (1965) 383-392.

[11] J. Frehse, Remarks on diagonal elliptic systems, in Partial differential equations and calculus of variations. Springer, Berlin (1988) 198-210.

[12] J. Frehse, Bellman Systems of Stochastic Differential Games with three Players in Optimal Control and Partial Differential Equations, edited by J.L. Menaldi, E. Rofman and A. Sulem. IOS Press (2001).

[13] S. Hildebrandt and K.-O. Widman, Some regularity results for quasilinear elliptic systems of second order. Math. Z. 142 (1975) 67-86.

[14] J. Leray and J.-L. Lions, Quelques résultats de Višik sur les problèmes elliptiques nonlinéaires par les méthodes de Minty-Browder. Bull. Soc. Math. France 93 (1965) 97-107.

[15] O.A. Ladyženskaja, V.A. Solonnikov and N.N. Ural'ceva, Linear and quasilinear equations of parabolic type. American Mathematical Society, Providence, R.I. (1967).

[16] M. Struwe, On the Hölder continuity of bounded weak solutions of quasilinear parabolic systems. Manuscripta Math. 35 (1981) 125-145.

[17] M. Wiegner, Ein optimaler Regularitätssatz für schwache Lösungen gewisser elliptischer Systeme. Math. Z. 147 (1976) $21-28$. 